\title{
Impacto socioeconómico de la variabilidad climática en pesca y turismo: antecedentes y propuesta metodológica
}

\author{
Mary L. Moreno-Díaz ${ }^{1}$ \\ 1. Centro Internacional en Política Económica para el Desarrollo Sostenible (CINPE) de la Universidad Nacional, \\ Heredia, Costa Rica; mmoreno@una.cr
}

Recibido 13-III-2019. Corregido 20-VIII-2019. Aceptado 18-X-2019.

\begin{abstract}
Socioeconomic impact of climate variability in fisheries and tourism: background and methodological proposal. Introduction: Climate variability has important effects on the different economic activities that take place in marine areas. Currently, there are no comprehensive methodologies to carry out an assessment process that consider all the variables and interactions between them. Objective: To propose a methodological approach that comprehensively includes the different stages in the process of economic valuation of ecosystem services in coastal areas, in the face of climate variability. Methods: A bibliographic review, expert consultations and an application of a methodological example using the impact over shark diving activities at Isla del Coco were conducted. Results: The proposed methodological approach begins with the socioeconomic and environmental characterization of the activity that uses a natural resource as input, followed by the identification and characterization of the environmental variables that affect the natural resource and of the effects that the variation of this resource has on the economic activity. Conclusions: The variables that make up the climate system, which relate to natural resources and economic activities, being influenced by external phenomena, produce effects that must be analyzed by comprehensive methodological approximations, within which one of the steps is the valuation methodology, that will allow generating policy recommendations that help to minimize these effects.
\end{abstract}

Key words: Climate Variability; Economic Valuation; Climatic System; Fishery; Tourism; Cocos Island.

Moreno-Diaz, M. L. (2020). Impacto socioeconómico de la variabilidad climática en pesca y turismo: antecedentes y propuesta metodológica. Revista de Biología Tropical, 68(Supl. 1), $\mathrm{S} 18-\mathrm{S} 32$.

La variabilidad del clima se refiere a variaciones en las condiciones climáticas medias y otras estadísticas del clima (como las desviaciones típicas, los fenómenos extremos, etc.), que pueden suceder en cualquier momento y en cualquier sitio del planeta y que genera un comportamiento anormal del clima, pero es un fenómeno temporal y transitorio (IPCC, 2001). La variabilidad puede deberse a procesos naturales internos que ocurren dentro del sistema climático (variabilidad interna), o a variaciones en el forzamiento externo natural o antropógeno (variabilidad externa). La Convención
Marco de Cambio Climático distingue entre 'cambio climático' atribuido a actividades humanas que alteran la composición atmosférica y 'variabilidad climática' atribuida a causas naturales (IPCC, 2001).

En los últimos años se ha generado gran cantidad de literatura relacionada con diferentes tipos de aproximaciones que permitan analizar la inestabilidad y sensibilidad del sistema climático y sus efectos generales sobre el planeta (Wigley \& Raper, 2001; Knutti, Stocker, Joos, \& Plattner, 2002; Murphy et al., 2004; Annan \& Hargreaves, 2006; Forest, Stone \& Sokolov, 
2006; Meinshausen 2006; Roebeling, Costa, Magalhaes-Filho, \& Tekken, 2013; Kuhfuss, Rey-Valette, Sourisseau, Heurtefeux, \& Rufray, 2016; Melo et al., 2017). Estos estudios evidencian que para aproximar los impactos del cambio y la variabilidad climática sobre la tierra en general y en actividades específicas como pesca y turismo, se deben analizar diferentes tipos de interacciones y retroalimentaciones de los componentes del sistema climático.

El objetivo del presente artículo es proponer un marco metodológico que contribuya a realizar un análisis integral para una adecuada valoración económica de los impactos del cambio climático sobre actividades productivas, teniendo en cuenta las relaciones entre las diferentes variables naturales, ambientales, sociales y económicas que intervienen.

\section{MATERIALES Y MÉTODOS}

En un artículo previo (Moreno-Díaz, 2015), se desarrolló una revisión bibliográfica analítica sobre las investigaciones que se han desarrollado tanto a nivel mundial como a nivel de los países de América Latina, para valorar los impactos de la variabilidad climática sobre la pesca y el turismo y se encontró que no existía un marco metodológico integral para valorar económicamente los efectos de variaciones en variables climáticas sobre actividades económicas. En el presente artículo se complementó con documentos más actualizados sobre los efectos que reporta la literatura de la variabilidad y el cambio climático sobre las actividades económicas y recursos naturales en zonas costeras, y se realizó una revisión de los diferentes sistemas de valoración económica que podrían emplearse para estos temas. Finalmente, con base en la información anterior, se platea una aproximación metodológica para tal fin y se presenta un ejemplo de aplicación de esta.

\section{RESULTADOS}

Impactos del cambio climático en las actividades económicas y recursos naturales en Zonas Costeras: Los procesos dinámicos naturales sufridos en muchas zonas costeras hacen difícil de distinguir estos procesos de aquellos atribuibles a la variabilidad y al cambio climático (Pirazzoli, Regnauld, \& Lemasson, 2004; Regnauld, Pirazzoli, Morvan \& Ruz, 2004; Cooper \& Navas, 2004). El poder determinar qué efectos sobre los recursos costeros son debidos a procesos naturales y cuales a la variabilidad y el cambio climático es todo un reto. Las oscilaciones climáticas relacionadas con la atmósfera y el océano pueden producir cambios costeros naturales (Viles \& Goudie, 2003). El fenómeno conocido como el Niño-Oscilación del Sur (ENOS), que se produce con una periodicidad promedio entre dos y siete años, es una de estas oscilaciones. Alfaro y Amador $(1996,1997)$ y Timmermann et al. (2018), definen este fenómeno como una corriente cálida que modifica la temperatura superficial del mar, usualmente los primeros meses del año y puede ocasionar impactos negativos sobre las zonas costeras como disminución de los recursos pesqueros y cambios severos en la morfología costera (Lizano, 1997; Lizano \& Salas, 2001).

Otros impactos estudiados han sido sobre i) la morfología de las playas como sucedió en Australia (Ranasinghe, McLoughlin, Short, \& Symonds, 2004); ii) la erosión en acantilados como es el caso de la costa central de California en los Niños de 1982-1983 y 1997-1998 (Stortlazzi \& Griggs, 2000); iii) la disminución de los niveles de agua de los Ecosistemas de Manglar como fue el caso de Australia (Rogers \& Saintilan, 2008), iv) la erosión costera, como el caso de la región de Languedoc-Rosellón, antigua región administrativa de Francia (Roebeling et al., 2013).

También es importante destacar que hay efectos negativos sobre los recursos costeros causados no solo por la gran presión de las poblaciones que viven en estas zonas y las actividades que se desarrollan en ellas, sino además por la gran contaminación que se presenta en las cuencas altas y medias y por la falta de planificación del componente urbano (Graham, 2008). En muchas zonas costeras también 
se realiza el drenaje de zonas de manglar, deforestación, disposición de desechos sólidos y líquidos y de fertilizantes. Según algunos autores (Scavia et al, 2002; Lotze et al., 2006) los impactos directos causados por el hombre han sido más significativos en los últimos años que aquellos que han sido atribuidos al cambio climático. Estudios recientes muestran algunos de estos efectos: i) la presencia de metales en recursos costeros por sobre la cantidad natural como en Chile (Valdés \& Sifeddine, 2009) y ii) los efectos de la explotación petrolera (García-Cuellar, Arrenguin-Sánchez, Hernández, \& Lluch-Costa, 2004).

Algunos de los impactos socioeconómi$\cos$ que han sido atribuidos a la variabilidad y al cambio climático en zonas costeras se presentan en el Tabla 1. En el caso de los recursos de agua dulce, Hay y Mimura (2006) analizron que una de las influencias directas de aumentos en el nivel del mar sobre los recursos hídricos en zonas costeras, es la intrusión del agua de mar en las aguas superficiales y los acuíferos costeros y la usurpación de aguas de marea en los estuarios y los sistemas rivereños costeros. Estos procesos producen también salinización de los ecosistemas de manglar (Allison, Khan, Goodbred, \& Kuehl, 2003). Estos impactos pueden tener efectos devastadores en algunas áreas debido a factores naturales y socioeconómicos propios que aumentan los niveles de riesgo. Aun cuando las zonas costeras mantienen gran proporción de la población mundial la oferta de agua en estas zonas es pequeña haciendo que estos efectos del cambio climático sean críticos (Nicholls, Wong, Burkett, Codignotto, \& Hay, 2007). A pesar de lo anterior, si el cambio climático aumenta la precipitación en zonas costeras esto permite el aumento en los niveles de aguas.

Se espera que el impacto de la variabilidad y el cambio climático en el sector pesquero se vea reflejado tanto en aquellas especies que se desarrollan en las zonas costeras como aquellas que se encuentran en alta mar o pelágicas (Hennessy et al., 2007). Algunos de esos impactos serán: cambios en las temperaturas del mar, corrientes, vientos, oferta de nutrientes, acidificación y lluvias. Se prevé que dentro de las variables biológicas se presenten impactos en: i) distribución y abundancia de aquellas especies impactadas, ii) composición de las comunidades; iii) estructura y dinámica de las comunidades incluyendo productividad. Con el aumento del nivel del mar aumenta la intrusión marina afectando las pesquerías costeras y los sitios que son criaderos para especies comerciales (Schallenberg, Hall, \& Burns, 2003).

El cambio y la variabilidad climática afectan los asentamientos y la infraestructura

TABLA 1

Resumen de los impactos socioeconómicos relacionados con el cambio climático en zonas costeras. Fuente: Nicholls et al., 2007.

TABLE 1

Summary of socio-economic impacts related to climate change in coastal areas. Source: Nicholls et al., 2007.

\begin{tabular}{lccccccc}
\hline $\begin{array}{c}\text { Sector } \\
\text { socioeconómico }\end{array}$ & $\begin{array}{c}\text { Aumento en } \\
\text { temperatura } \\
\text { (aire y mar) }\end{array}$ & $\begin{array}{c}\text { Eventos } \\
\text { (xtremos } \\
\text { tormentas } \\
\text { y olas) }\end{array}$ & $\begin{array}{c}\text { Inundaciones } \\
\text { (nivel del } \\
\text { mar y } \\
\text { escorrentía) }\end{array}$ & $\begin{array}{c}\text { Aumento en } \\
\text { nivel de mantos } \\
\text { acuíferos } \\
\text { (nivel del mar) }\end{array}$ & $\begin{array}{c}\text { Erosión } \\
\text { (nivel del mar, } \\
\text { tormentas } \\
\text { y olas) }\end{array}$ & $\begin{array}{c}\text { Intrusión de } \\
\text { agua salada } \\
\text { (nivel del mar, } \\
\text { escorrentía) }\end{array}$ & $\begin{array}{c}\text { Efectos } \\
\text { biológicos (todos } \\
\text { los forzantes } \\
\text { climáticos) }\end{array}$ \\
\hline Recursos de agua dulce & $\mathrm{X}$ & $\mathrm{X}$ & $\mathrm{X}$ & $\mathrm{X}$ & - & $\mathrm{X}$ & $\mathrm{X}$ \\
Agricultura y forestal & $\mathrm{X}$ & $\mathrm{X}$ & $\mathrm{X}$ & $\mathrm{X}$ & - & $\mathrm{X}$ & $\mathrm{X}$ \\
Pesca y acuicultura & $\mathrm{X}$ & $\mathrm{X}$ & $\mathrm{X}$ & - & $\mathrm{X}$ & $\mathrm{X}$ & $\mathrm{X}$ \\
Salud & $\mathrm{X}$ & $\mathrm{X}$ & $\mathrm{X}$ & $\mathrm{X}$ & - & $\mathrm{X}$ & $\mathrm{X}$ \\
Recreación y turismo & $\mathrm{X}$ & $\mathrm{X}$ & $\mathrm{X}$ & - & $\mathrm{X}$ & - & $\mathrm{X}$ \\
Biodiversidad & $\mathrm{X}$ & $\mathrm{X}$ & $\mathrm{X}$ & $\mathrm{X}$ & $\mathrm{X}$ & $\mathrm{X}$ & $\mathrm{X}$ \\
Infraestructura & $\mathrm{X}$ & $\mathrm{X}$ & $\mathrm{X}$ & $\mathrm{X}$ & $\mathrm{X}$ & $\mathrm{X}$ & - \\
\hline
\end{tabular}


costera de varias maneras: el aumento en el nivel del mar y en la intensidad de las precipitaciones puede provocar inundaciones, además la intrusión salina afecta no solo las construcciones sino la calidad de las aguas subterráneas. La erosión producida por fenómenos como las tormentas es también un problema grave en estas zonas, ya que permite que el mar gane terreno sobre las construcciones (Lizano \& Salas, 2001). La costa este de los Estados Unidos ha sido muy impactada por las tormentas y aunque no se han podido establecer tendencias e intensidades en el largo plazo, el aumento en el nivel del mar ha intensificado los daños a estructuras causados por tormentas fuertes en este siglo.

La pérdida de recursos costeros como ecosistemas de manglar y otras barreras naturales ponen al descubierto las poblaciones y las construcciones como puentes y caminos haciéndolos vulnerables a eventos extremos como tormentas y al aumento en el nivel del mar. Muchos países en Europa (Holanda) y este de Asia tienen defensas artificiales costeras contra los fenómenos climáticos como inundaciones y erosión (Jonkman, Stive, \& Vrijling, 2005), estas construcciones y los asentamientos urbanos que protegen también son vulnerables a los efectos del cambio climático.

En el caso de la biodiversidad son diversos los ecosistemas costeros que se pueden ver afectados por salinización, inundaciones y otros provocados por los tormentas, huracanes y aumentos en el nivel del mar. En el caso de la muerte de las larvas (de las que depende el coral) y la segmentación de los arrecifes de coral como consecuencia del cambio climático, Munday et al. (2009) concluyen que no hay suficiente certeza sobre estos efectos como para realizar predicciones robustas.

Los ecosistemas de manglar también se ven afectados por el cambio climático, aunque no existen aún estudios al respecto, se sabe que estos efectos seguramente reforzarán aquellos causado por el hombre al convertirlos en camaroneras. Casos de la conversión de estos ecosistemas son presentados para Vietnam (Binhh, Phillips, \& Demaine, 1997), Bangladesh e
India (Zweig, 1998), México (Contreras-Espinosa \& Warner, 2004) y Malasia (Ong, 2001) entre otros. En Centroamérica y debido al aumento en el nivel del mar se observarán consecuencias adversas en las zonas costeras bajas de El Salvador y en la disponibilidad de agua potable en la costa pacífico de Costa Rica. En cuanto al turismo costero, los países con mayores impactos serán aquellos en los que el PIB de este sector contribuya significativamente a la balanza de pagos y al empleo (este es el caso de Costa Rica) (CEPAL, 2009).

\section{Valoración Económica del cambio climático}

Diferentes sistemas de Valoración Económica: De acuerdo a Moreno-Díaz (2009), la base de cualquier marco metodológico sobre valoración económica de recursos naturales está basado en el concepto de Valor Económico Total (VET), que está conformado por el valor de uso y el de no uso y que ha sido tratado de diversas maneras por diferentes autores (Walsh, Loomis, \& Gillman, 1984; Azqueta-Oyarzun, 1994; Pearce \& Turner, 1995; Freeman, Herriges \& Kling, 2014), ya que su concepción teórica se adapta dependiendo de las necesidades de los investigadores. De esta forma, el valor asignado a los Recursos Naturales y Ambientales (RENA's), va a depender de su capacidad de generar bienes y servicios para satisfacer necesidades humanas. Como se observa (Fig. 1), el valor de uso hace referencia al consumo de los recursos naturales bien sea en forma directa, indirecta o en el futuro, y el de no uso al valor que pueden tener los RENA's para las generaciones futuras y el valor de conocimiento de su existencia continua.

Sharp y Batstone (2008) adaptaron el concepto del VET (Fig. 1), para el caso de los recursos naturales marino-costeros indicando que este proporciona un marco general de política para intentar maximizar el valor del flujo de servicios que provienen de los activos naturales marinos. El desarrollo de la aproximación metodológica se basa en la Teoría Neoclásica de la optimización que se emplea en la 


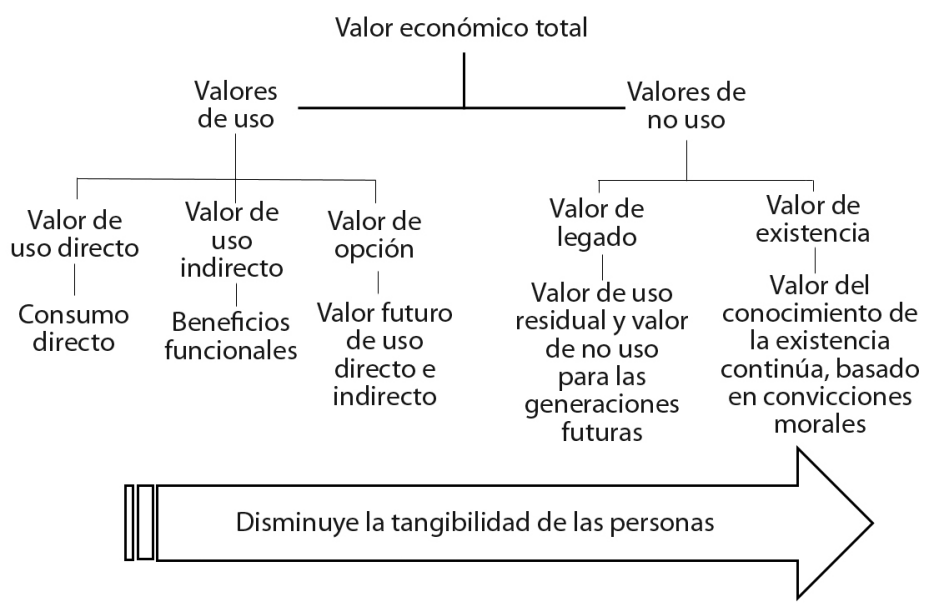

Fig. 1. Componentes del valor económico total. Fuente: Elaboración propia con base en Pearce y Turner (1995).

Fig. 1. Total economic value components. Source: Own elaboration based on Pearce and Turner (1995).

economía de recursos naturales. El proceso inicia con la distinción entre aquellos recursos que son renovables y aquellos que no lo son. Si se parte del supuesto de la maximización del VET o más específicamente del valor presente neto de los beneficios, se identifican los servicios que tienen precio en el mercado y aquellos que no (Fig. 2). En el caso en que no se cuente con precios de mercado se debe seleccionar uno de los métodos de no mercado con los que cuenta la economía neoclásica.

Los valores de uso y no uso de los bienes y servicios generados por los RENA's deben ser aproximados por medio de metodologías de valoración. En la literatura, se han estructurado estas metodologías y son clasificadas de distintas formas, dependiendo de lo que se quiera medir, del concepto de valor adoptado, los algoritmos de solución usados y el tipo de información requerido (Agüero, 1994). Una forma de clasificación es la que agrupa los métodos en directos, indirectos, de valoración contingente y de variables y modelos macroeconómicos.

Según Barzev (2002) los métodos directos se basan en precios de mercado disponibles o

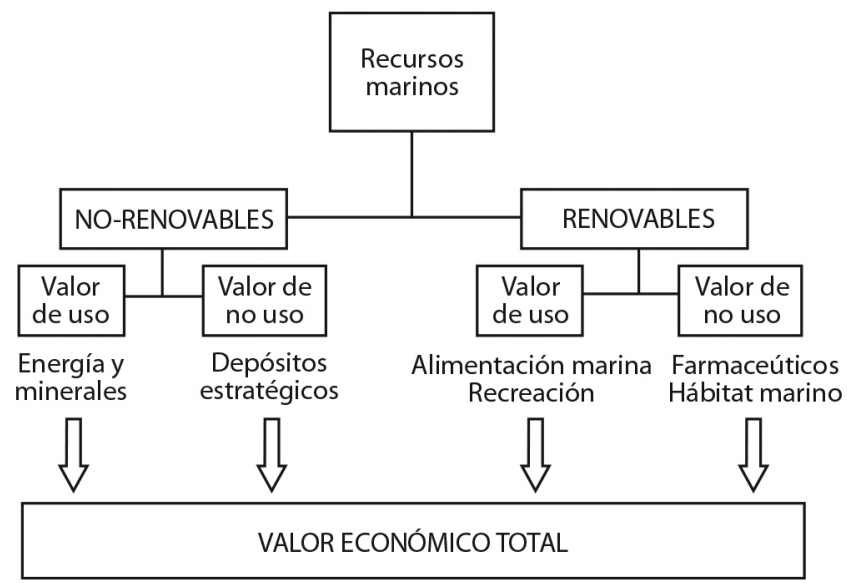

Fig. 2. Valor económico total de los recursos marinos. Fuente: Elaboración propia con base en Sharp y Batstone (2008).

Fig. 2. Total economic value of marine resources. Source: Own elaboration based on Sharp and Batstone (2008). 
en observación de cambios en la productividad y son aplicados cuando un cambio en la calidad del ambiente o disponibilidad de un recurso impacta la producción o la productividad. Se emplea como fuente de información, los parámetros de conductas observadas, como los precios pagados o gastos efectuados (según la clasificación de Munasinghe), reflejada en mercados convencionales. Por otro lado, y según el mismo autor, los métodos indirectos hacen uso de los precios de mercado en forma indirecta y son empleados cuando diversos aspectos o atributos de los recursos naturales o servicios ambientales no tienen precios reflejados en un mercado establecido. Finalmente menciona los métodos de Valoración Contingente y otros (Fig. 3). Es importante destacar que los cuatro tipos de metodologías (directos, indirectos, contingentes y de variables y modelos macroeconómicos) se sitúan en una perspectiva temporal diferente. Mientras que los métodos directos e indirectos intentan averiguar a través de la observación de su conducta el valor que la persona otorga a un cambio que ya ha ocurrido, el método de Valoración Contingente presenta, en general, una situación hipotética, que todavía no se ha dado (Azqueta-Oyarzun, 1994).

Antes de decidir cuál de las metodologías aplicar, se deben identificar los servicios que los RENA's prestan para luego seleccionar aquella que mejor se adapte. En el caso de los recursos marino-costeros, Wilson y Liu (2008), emplean un enfoque metodológico en el que los bienes y servicios costeros pueden dividirse en dos categorías: (1) la provisión de bienes y servicios de mercado como agua potable, transporte, generación de electricidad e irrigación y (2) la provisión de aquellos bienes y servicios de no mercado que incluye biodiversidad, soporte para ecosistemas terrestres y de estuario, hábitat para la existencia de plantas y animales y la satisfacción que les deriva a las personas el saber que las plantas y los corales existen. Los autores presentan una agrupación

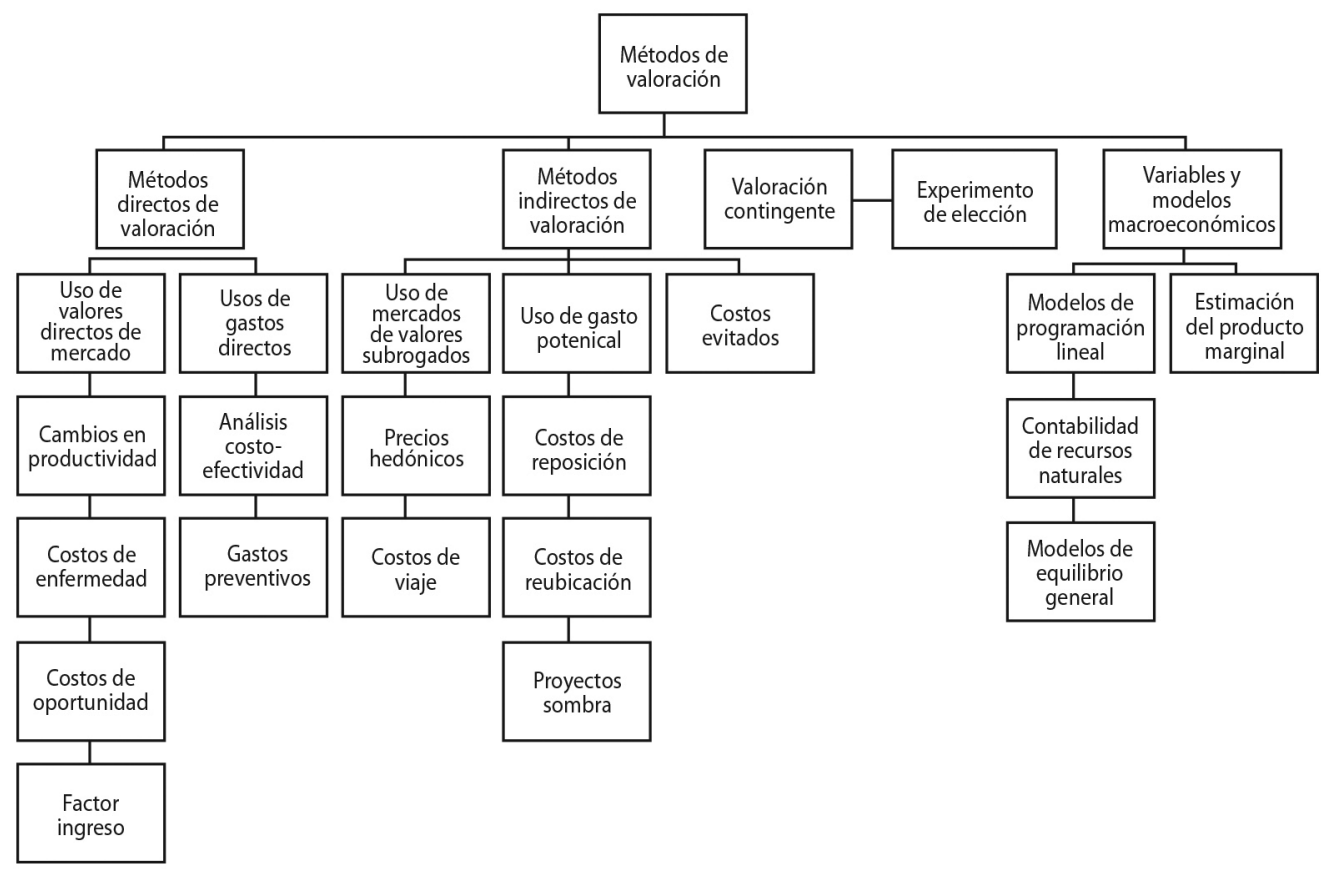

Fig. 3. Clasificación de las metodologías de valoración económica para Servicios Ecosistémicos. Fuente: Elaboración propia con base en Dixon, Scura, Carpenter \& Sherman (1994); Barzev (2002) y Wilson y Liu (2008).

Fig. 3. Classification of economic valuation methodologies for Ecosystem Services. Source: Own elaboration based on Dixon et al. (1994); Barzev (2002) and Wilson \& Liu (2008). 
de los servicios ecosistémicos marinos y costeros en cuatro categorías básicas de acuerdo con sus características funcionales:

Servicios de regulación. Los ecosistemas regulan esencialmente procesos ecológicos y sistemas de soporte de vida a través de ciclos biogeoquímicos y otros procesos biosféricos. Esto incluye aspectos como prevención de la perturbación y control de inundaciones.

Servicios culturales. Los ecosistemas contribuyen al mantenimiento de la salud humana y el bienestar proveyendo disfrute cultural, integridad histórica, recreación y estética.

Servicios de soporte. Los ecosistemas proveen un rango de servicios que son necesarios para la producción de otras tres categorías de servicios: ciclo de los nutrientes, formación de suelo y funciones de los hábitats.

Servicios de aprovisionamiento. La función de aprovisionamiento de los ecosistemas ofrece una larga variedad de bienes ecosistémicos mercadeables y otros servicios para consumo humano, desde alimento y materias primas hasta recursos energéticos.

Es importante resaltar que todos estos servicios se optimizan siempre y cuando los ecosistemas mantengan una integridad ecológica óptima. Para las tres primeras clasificaciones, los autores proponen emplear algunas de las metodologías de valoración presentadas (Fig. 3), adaptadas al caso de los recursos marino-costeros.

En los últimos años se han elaborado estudios relacionados con valoración de servicios ecosistémicos costeros, no necesariamente relacionados con variabilidad climática. Maldonado y Cuervo-Sánchez (2016) realizaron un estudio para aproximar el valor económico de la existencia del Parque Nacional Natural Corales de Profundidad en Colombia, para ello emplearon la metodología de Valoración Contingente y obtuvieron una estimación de 95 millones de dólares por año, lo compararon con el presupuesto que se le brinda al parque para su gestión y encontraron que es menor al $1 \%$ de la aproximación realizada. En el trabajo desarrollado por Cuervo, Maldonado, y Rueda (2014) se realiza una valoración de los cambios en los beneficios generados por los recursos pesqueros si se aumenta la cantidad de Áreas Protegidas y concluyen que estas áreas generan en el mediano plazo un aumento de los beneficios de la actividad pesquera.

Contreras (2016) desarrolló un estudio en el que se realiza una aproximación al valor de los servicios de soporte a la pesquería provisto por el ecosistema de manglar en la Ciénaga Grande de Santa Marta, empleando metodologías de precios de mercado y obtuvo una alta productividad de este ecosistema. En una línea temática similar, Arguedas (2015) realizó un trabajo de valoración basado en metodologías de precios de mercado de los servicios ecosistémicos que ofrece el manglar del Golfo de Nicoya en Costa Rica, y obtuvo que los principales beneficios se dan por la obtención de moluscos, secuestro de carbono y belleza escénica. Gómez (2017) realizó una valoración económica, resultante de la aplicación del método AMUVAN (Analytic MulticriteriaValuation Method), en la que aproximó que el valor que tienen para la comunidad costera de Safor (Comunidad Valenciana) los servicios ecosistémicos de aprovisionamiento, regulación, protección del hábitat y servicios culturales se encuentra en un intervalo de (120568 657 $€$ у $677654020 €)$.

Algunos estudios recientes sí incluyen los tres temas tratados en el presente artículo (valoración económica, variabilidad climática y zonas costeras). Roebeling et al. (2013), evaluaron las pérdidas en los servicios ecosistémicos por la erosión costera en Europa, revisando las tendencias históricas y realizando proyecciones futuras. Para el desarrollo del estudio utilizaron los siguientes métodos: transferencia de beneficios como método de valoración, intersecciones históricas de mapas de uso de la tierra costera y simulaciones de escenarios. Sobre los resultados se señala que durante el período 1975 a 2006, "disminuyeron de aproximadamente $€ 22.3$ mil millones por 
año en 1975 a aproximadamente $€ 21.6$ mil millones por año en 2006" (Roebeling et al., 2013 , p. 392). Respecto al período 2006-2050 se indica que "los valores disminuyeron de aproximadamente $€ 21.6$ mil millones por año en 2006 a entre $€ 20.1$ y $€ 19.4$ mil millones por año para 2050" (Roebeling et al., 2013, p. 393).

Otro estudio realizado en estos temas es el de Kuhfuss et al. (2016), quienes desarrollaron el estudio en la región de Languedoc-Rosellón, antigua región administrativa de Francia, examinando las consecuencias de un incremento de unn metro en el nivel del mar para los humedales que están en peligro de inmersión. A nivel metodológico, para estimar el impacto económico de las ganancias y pérdidas en los servicios se utilizaron varios métodos dependiendo de las características de los servicios y la información disponible. Entre los métodos utilizados se encuentran: precios de mercado, valor de transferencia y disponibilidad a pagar considerando el valor de transferencia. Los resultados indicaron que lo obtenido actualmente, y que se perdería con el incremento del mar, equivale a 14 euros/ha/año en los servicios de provisión, 710 euros/ha/año en los servicios de regulación, 243 euros/ha/año por los servicios de soporte, 2700 euros por cazador y 0.24 euros/casa/año en los servicios culturales y recreacionales, para un total de 3783, 24 euros.

Melo et al. (2017), estimaron los efectos económicos (impacto) de futuras sequías en Colombia a través de los costos económicos incurridos en el país a partir del fenómeno El Niño en 2015; emplearon metodologías de precios de mercado y obtuvieron que los costos económicos de eventos de variabilidad climática similares al analizado, reducirían como mínimo el equivalente al $0.7 \%$ del PIB si el país no toma las medidas necesarias para adaptarse al cambio climático.

Propuesta metodológica: Como se ha detallado en los apartados anteriores, las relaciones entre las variables del sistema climático no son unidireccionales, es decir, no siempre se puede evidenciar una causa-efecto directos. Los efectos pueden verse en varios sentidos y pueden ser indirectos también. En términos de las relaciones entre variables ambientales y económicas, los modelos que han sido desarrollados para establecer los escenarios climáticos parten del supuesto que la actividad económica genera una serie de gases de efecto invernadero que tienen implicaciones sobre variables climáticas como la temperatura y la precipitación y que afectan a su vez algunos recursos naturales y a actividades económicas. La magnitud y medición de los efectos físicos causados por el aumento de gases de efecto invernadero sobre las variables climáticas son medidas por expertos climatólogos por medio de Modelos de Circulación General (MCG) y los escenarios de emisiones del IPCC. Los MCG son representaciones numéricas tridimensionales, que se emplean para simular el comportamiento futuro del sistema climático global. Se han realizado esfuerzos también en generar datos sobre las variables climáticas a nivel regional, empleando como base los modelos globales (Echeverría, 2004; Alvarado, 2006).

Los resultados del desarrollo y combinación de modelos permiten obtener escenarios climáticos en donde se especifica los cambios en las variables climáticas como la temperatura media anual y la precipitación para determinado escenario de emisiones. Debido a que en el presente artículo se desarrolla un esfuerzo por analizar los efectos de las variables climáticas sobre los recursos naturales y las actividades económicas que los emplean en zonas costeras, la propuesta se basa en que el proceso de análisis se inicie identificando la actividad que se desea estudiar y de esta manera analizar en primera instancia, las diferentes variables climáticas y recursos naturales que forman parte de la actividad.

A continuación, con base en las relaciones y conceptos analizados en las secciones anteriores se presenta una propuesta metodológica de siete pasos para la valoración de los efectos de la variabilidad climática sobre actividades desarrolladas en zonas costeras que emplean recursos naturales. En la Fig. 4 se aprecia el proceso de análisis que permite identificar y valorar los efectos que sobre una actividad 


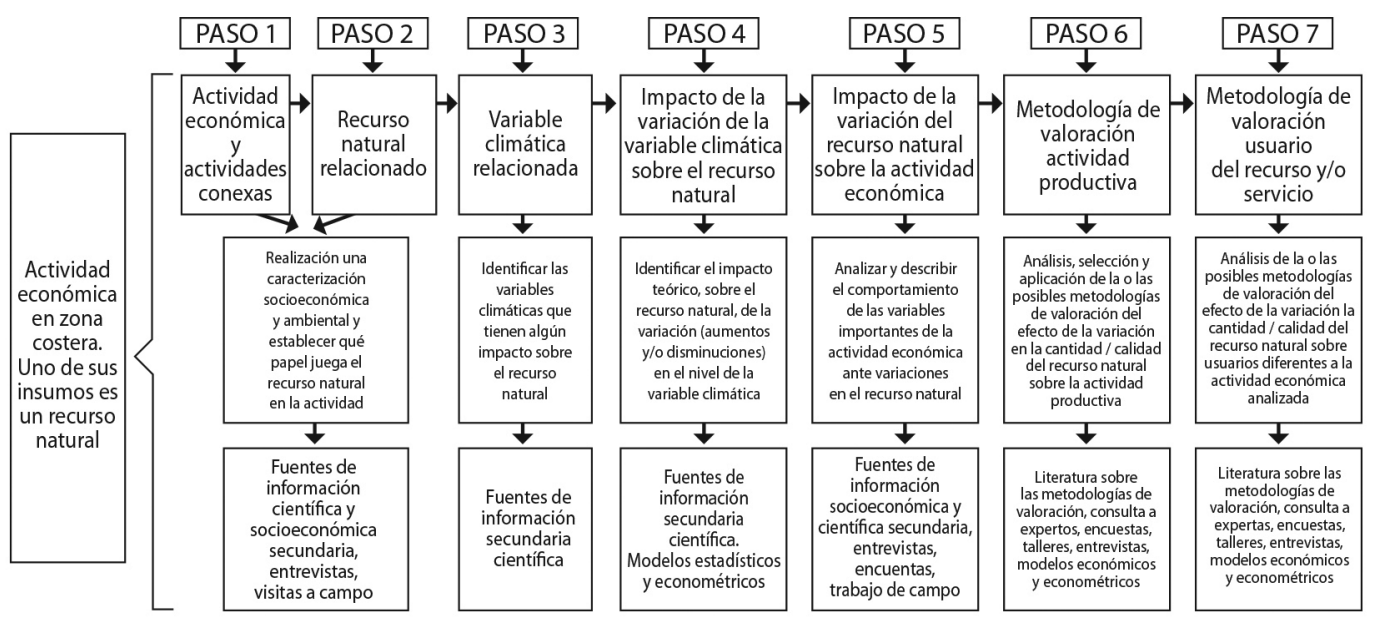

Fig. 4. Propuesta metodológica para valorar los efectos de la variabilidad climática sobre una actividad económica costera. Fuente: Elaboración propia.

Fig. 4. Methodological proposal to assess the effects of climate variability on a coastal economic activity. Source: Own elaboration.

económica pueden tener variaciones en las variables climáticas.

Paso 1. La actividad económica costera identificada, que en el caso de Costa Rica puede ser pesca o turismo (Moreno-Díaz, 2012), debe ser caracterizada en una primera instancia en cuanto a tipos de recursos naturales que emplea, cantidad de empleo que genera, zonas donde se desarrolla y si hay otras actividades (conexas) que se desarrollan amparadas en esta actividad. Las actividades conexas tienen en su función de producción uno o varios recursos naturales como insumos. Se debe desarrollar una caracterización socioeconómica y ambiental que permita establecer qué papel juega el recurso natural tanto en la actividad productiva general como en las actividades conexas.

Paso 2. Es importante determinar la relevancia del recurso natural en el desarrollo de la actividad económica y la forma en que puede medirse (si disminuye el recurso, disminuyen los ingresos). Lo anterior se puede realizar empleando fuentes de información científica y socioeconómica secundaria, entrevistas y visitas de campo. En general es deseable tener series de datos sobre el desarrollo de las actividades económicas, sin embargo, en países como Costa Rica la cultura de la sistematización de la información en actividades costeras no está desarrollada.

Paso 3. Una vez realizada la caracterización de la actividad económica, teniendo en cuenta aspectos relevantes propios de la actividad y el uso de los recursos naturales, se deben identificar las variables climáticas que tienen influencia sobre estos recursos naturales.

Paso 4. Posteriormente se debe realizar un análisis del impacto teórico que tendría sobre el recurso natural, la variación (aumentos y/o disminuciones) en el nivel de la variable climática, es decir lo equivalente a una Función Dosis-respuesta; este tipo de análisis debe ser hecho con base en información secundaria desarrollada por científicos especialistas en el tema (climatólogos y biólogos, entre otros), de ser posible además se deben realizar entrevistas con estos científicos para entender mejor las relaciones e impactos identificados.

Paso 5. Una vez identificadas las posibles variaciones en el recurso natural debidas a cambios en la variable ambiental es necesario 
determinar cuál será el impacto de la variación del recurso natural sobre la actividad económica. Para este fin se debe analizar y describir el comportamiento de las variables importantes de la actividad económica y como estas son afectadas por oscilaciones en el recurso natural.

Paso 6. Con la información obtenida en los procesos anteriores se debe analizar, seleccionar y aplicar la o las metodologías de valoración del efecto de la variación en la cantidad/ calidad del recurso natural sobre la actividad productiva. En este paso es necesario realizar una revisión de literatura sobre las metodologías, consultas a expertos, talleres, encuestas, entrevistas y revisión de modelos económicos y econométricos dependiendo de la metodología o metodologías que se seleccionen.

Paso 7. Aquí es muy importante tener presente que no sólo se deben aplicar metodologías para aproximar el impacto de la variación en el recurso natural sobre la actividad productiva, sino que también existen un grupo de metodologías de valoración económica que permite aproximar el efecto que estas variaciones conllevan para los usuarios del recurso y/o servicio que él brinda.

Una vez desarrollado el proceso metodológico, se deben realizar sugerencias sobre cuáles deben ser los cursos de acción a nivel de políticas y estrategias para mitigar el efecto que puede tener el cambio/variabilidad climática sobre los recursos naturales y las actividades económicas que dependen de ellos.
Ejemplo de aplicación de la metodología sugerida: A continuación, se realiza un planteamiento de cómo se debería desarrollar la metodología sugerida (Fig. 5); también se puede encontrar más detalle en MorenoDíaz (2012).

El problema que se desea analizar es el efecto que tienen los cambios en las variables climáticas sobre actividades productivas que se desarrollan alrededor de la Isla del Coco. En primera instancia, se realizó una caracterización socioeconómica lo más completa posible en la que se describe detalladamente la forma en que se desarrolla la actividad turistica, qué servicios se ofrecen, quién los ofrece y quiénes los demandan.

Para este ejemplo se empleó una de las actividades conexas al turismo, las empresas navieras que llevan turistas a la Isla del Coco. Para este caso se identificó el tiburón martillo (Sphyrna lewini) como el recurso natural del cual depende la actividad conexa. Del análisis realizado se determinó que la variable climática más importante en este caso fue la temperatura superficial del mar, ya que cuando aumenta causa la disminución en la cantidad de avistamientos de tiburón martillo. Esta disminución podría causar a su vez que menos turistas visiten la Isla, provocando eventualmente una disminución de aproximadamente $30 \%$ en los ingresos de las empresas navieras. Se propone el desarrollo de una metodología de valoración de mercado para cuantificar este efecto. Además, se emplearon metodologías de preferencias declaradas para analizar el cambio en las

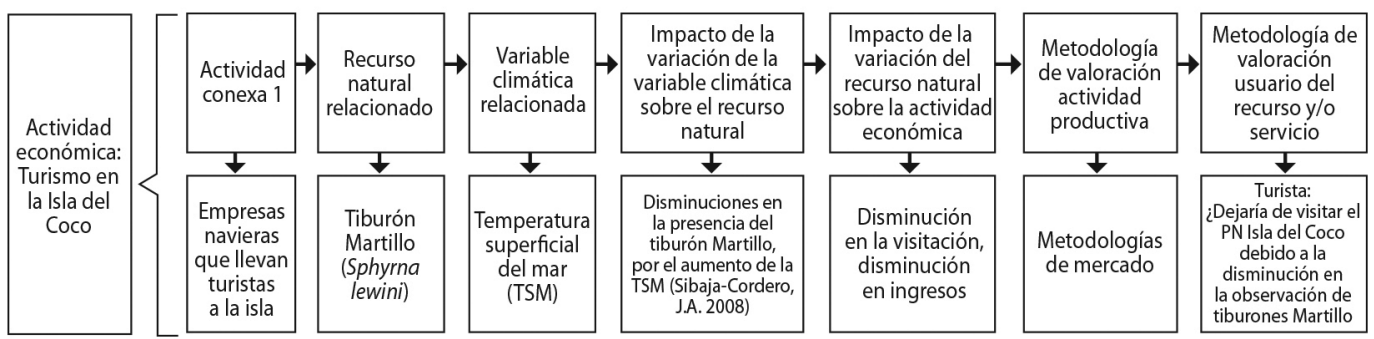

Fig. 5. Ejemplo de aplicación de la propuesta metodológica para valorar los efectos de la variabilidad climática sobre una actividad turística. Fuente: Moreno-Díaz (2012).

Fig. 5. Example of application of the methodological proposal to assess the effects of climate variability on the tourist activity. Source: Moreno-Díaz (2012). 
preferencias de los turistas si se presenta un cambio en la disponibilidad del recurso natural.

\section{DISCUSIÓN}

Las variables que conforman los componentes del sistema climático, al ser influenciadas por fenómenos externos, pueden variar de formas diferentes y esto provoca que el cambio climático sea más difícil de predecir. Sin embargo, estas variaciones han sido medidas por diferentes grupos de especialistas, que han estructurado series de datos físicas como la temperatura superficial del mar, la precipitación y otros fenómenos meteorológicos que permiten pronosticar los efectos que podrían llegar a tener este cambio en los diferentes ámbitos. La valoración económica de estos efectos, considerando los temas de valoración económica, ha sido diversa ya que se han empleado, o bien un grupo de metodologías que permiten aproximar el efecto inmediato del impacto, u otro grupo que desarrolla modelos macroeconómicos generales que analiza efectos sobre todo el sistema macroeconómico de cambios en una de las variables ambientales.

En el primer caso se obtiene una aproximación numérica del impacto (Tol, 2007; Raschky, 2008), pero no se realiza una sistematización que permita la organización de una serie de pasos para la aplicación de métodos científicos en las diferentes áreas que involucran estos impactos, es decir, un análisis exhaustivo del comportamiento de los recursos naturales impactados y su relación con las variables climáticas y sus efectos sobre las actividades socioeconómicas dependientes de estos recursos. Por lo anterior, además, no se pueden hacer sugerencias para la mitigación de impactos causados por la variabilidad y el cambio climático. En el segundo caso en donde se emplean modelos macroeconómicos (Melo et al., 2017), las estimaciones pueden subvalorar los efectos reales que sobre una actividad económica puede tener una variación en el stock o calidad del recurso natural, no se hace un análisis detallado metodológico para analizar los efectos de la variabilidad climática.
Los efectos socioeconómicos causados por la variabilidad y el cambio climático en las zonas costeras deben ser aproximados y analizados con base en una metodología integradora que permita incluir todas aquellas variables que afectan el desarrollo de la actividad económica que se esté analizando. En este sentido el marco metodológico que se propone en el presente artículo realiza un proceso analítico no solo de la actividad económica como tal sino de las variables relacionadas con los recursos naturales que se emplean y de cómo las variables climáticas afectan estos recursos que a su vez afectan los ingresos de la actividad económica, incluyendo además no solamente especialista en valoración sino también especialistas científicos y sociales que permitan incorporar adecuadamente los efectos analizados.

Las experiencias recientes existentes de la integración de la valoración económica, la variabilidad climática y las zonas costeras (Roebeling et al., 2013; Kuhfuss et al., 2016), aunque involucran algunos de los aspectos presentados en el presente artículo, no se desarrollan bajo una aproximación metodológica integral que permita evidenciar claramente las interrelaciones entre los diferentes componentes económicos, ambientales y sociales para al final obtener la aproximación al valor de los efectos de la variabilidad climática sobre las actividades económicas usuarias de servicios ecosistémicos en zonas costeras.

Con los resultados obtenidos en la aplicación de la metodología sugerida en el presente artículo es posible generar recomendaciones para que la política ambiental hacia las actividades y sectores analizados sea más eficiente.

\section{CONCLUSIONES}

En el artículo se enfatiza que las variables que conforman el sistema climático, que tienen relación con los recursos naturales y las actividades económicas, al ser influenciadas por fenómenos externos, producen efectos que deben ser analizados por medio de metodologías de valoración económica, lo que permite generar recomendaciones de política 
que contribuyan a minimizar esos efectos. En la revisión bibliográfica desarrollada se encontró, que estos efectos han sido valorados económicamente con metodologías que no tienen en cuenta la diversidad de aspectos que deben incluirse en estos procesos de manera integral. Por lo anterior, se propone un marco metodológico que tiene en cuenta las variables climáticas, de recursos naturales y las actividades socioeconómicas que se ven afectadas por variaciones del sistema climático propias del cambio climático.

El marco metodológico propuesto se aplicó a un estudio de caso en el Parque Nacional Isla de Coco, en donde se obtuvo la temperatura superficial del mar como la variable climática más importante en donde sus variaciones generan menos cantidad de avistamientos de tiburones martillos (Sphyrna lewini), lo que provoca disminución en la visitación y a su vez menores ingresos tanto de las empresas navieras como de todas las actividades relacionadas con esta actividad.

El análisis integral de los efectos del cambio climático, generado con la metodología sugerida permite generar recomendaciones de política que pueden contribuir en la toma de decisiones para la adaptación al cambio climático para diferentes actores de la sociedad.

Declaración de ética: la autora declara que está de acuerdo con esta publicación; que no existe conflicto de interés de ningún tipo; y que ha cumplido con todos los requisitos y procedimientos éticos y legales pertinentes. Todas las fuentes de financiamiento se detallan plena y claramente en la sección de agradecimientos. El respectivo documento legal firmado se encuentra en los archivos de la revista.

\section{AGRADECIMIENTOS}

Este trabajo es parte del trabajo doctoral de la autora dentro del Doctorado en Ciencias Naturales para el Desarrollo (DOCINADE), programa conjunto de la Universidad Nacional (UNA), Costa Rica, Instituto Tecnológico de Costa Rica (TEC) y la Universidad Estatal a
Distancia (UNED), Costa Rica. Parte de la información aquí consignada fue elaborada por la autora en el marco del Programa integrado de análisis de Políticas Públicas para la gestión sostenible de los Recursos Naturales y Servicios Ambientales en Costa Rica (Códigos 0170-12, 055103, NGEH01) y del Proyecto de Valoración Económica de Servicios Ecosistémicos: Análisis de la implementación de los lineamientos en Costa Rica (0196-17, 025508, IAIg06) de la Universidad Nacional, Costa Rica.

\section{RESUMEN}

Introducción: La variabilidad climática tiene efectos importantes sobre las diferentes actividades económicas que se desarrollan en zonas costeras y que emplean servicios ecosistémicos para su funcionamiento. Actualmente, no existen metodologías integrales que permitan realizar un proceso de valoración teniendo en cuenta todas las variables y las interacciones entre ellas. Objetivo: Proponer una aproximación metodológica que incluya de manera integral las diferentes etapas en el proceso de valoración económica de servicios ecosistémicos en zonas costeras, ante la variabilidad climática. Métodos: Se realizó una revisión bibliográfica, consulta a expertos y se empleó un ejemplo de aplicación usando la actividad turística de buceo con tiburones martillos en la Isla del Coco. Resultados: La aproximación metodológica propuesta inicia con la caracterización socioeconómica y ambiental de la actividad que emplea el recurso natural como insumo, posteriormente se realiza una identificación y caracterización de las variables ambientales que afectan el recurso natural y los efectos que la variación de este recurso tiene sobre la actividad económica. Conclusiones: Las variables que conforman el sistema climático, que tienen relación con los recursos naturales y las actividades económicas, al ser influenciadas por fenómenos externos, producen efectos que deben ser analizados por medio de aproximaciones metodológicas integrales, dentro de las cuales uno de los pasos es la metodología de valoración, que permitan generar recomendaciones de política que contribuyan a minimizar esos efectos.

Palabras Clave: Variabilidad climática; valoración económica; sistema climático; pesca; turismo; Isla del Coco.

\section{REFERENCIAS}

Agüero, M. (1994). Valoración social y económica de manglares: problemas y métodos. El método de los coeficientes integrales. Revista Faro, 1, 17-21. 
Alfaro, E., \& Amador, J. (1996). El Niño-Oscilación del Sur y algunas series de temperatura máxima y brillo solar en Costa Rica. Tópicos Meteorológicos y Oceanográficos, 3(1), 19-26.

Alfaro, E., \& Amador, J. A. (1997). Variabilidad y cambio climático en algunos parámetros sobre Costa Rica y su relación con fenómenos de escala sinóptica y planetaria. Tópicos Meteorológicos y Oceanográficos, 4(1), 51-62.

Alvarado, L. (2006). Escenarios de Cambio Climático para Centroamérica. Proyecto: AIACC-LA06. SICA, CRRH, UCR. (Documento Técnico, 25p.).

Allison, M. A., Khan, S. R., Goodbred Jr, S. L., \& Kuehl, S. A. (2003). Stratigraphic evolution of the late Holocene Ganges-Brahmaputra lower delta plain. Sedimentary Geology, 155(3-4), 317-342.

Annan, J. D., \& Hargreaves, J. C. (2006). Using multiple observationally based constraints to estimate climate sensitivity. Geophysical Research Letters, 33, L06704

Arguedas, M. (2015). Valoración económica de servicios ecosistémicos brindados por el manglar del Golfo de Nicoya, Costa Rica. (Tesis de maestría). Centro Agronómico Tropical de Investigación y Enseñanza CATIE, Turrialba, Costa Rica.

Azqueta-Oyarzun, D. (1994). Valoración económica de la calidad ambiental. Madrid, España: McGraw-Hill.

Barzev, R. E. (Ed.) (2002). Guía metodológica de valoración económica de bienes, servicios e impactos ambientales. Un aporte para la gestión de ecosistemas y recursos naturales en el Corredor Biológico Mesoamericano. BM. Serie Técnica 04, Proyecto para la Consolidación del Corredor Biológico Mesoamericano. Managua, Nicaragua: Oficina Regional de Coordinación del Corredor Biológico Mesoamericano.

Binh, C. T., Phillips, M. J., \& Demaine, H. (1997). Integrated shrimp-mangrove farming systems in the Mekong delta of Vietnam. Aquaculture Research, 28(8), 599-610.

CEPAL (2009). Informe de Factibilidad: Economía del Cambio Climático en Centroamérica. LC/ MEX/L.897. México.

Contreras, A. (2016). Valoración económica del servicio ecosistémico de soporte a la pesquería provisto por el ecosistema de manglar en la Ciénaga Grande de Santa Marta. Economía del Caribe, 18, 3.

Contreras-Espinosa, F., \& Warner, B. G. (2004). Ecosystem characteristics and management considerations for coastal wetlands in Mexico. Hydrobiologia, 511, 233-245.
Cooper, J. A. G., \& Navas, F. (2004). Natural bathymetric change as a control on century-scale shoreline behaviour. Geology, 32, 513-516.

Cuervo, R., Maldonado, J. H., \& Rueda, M. E. (2014). Valoración de los servicios ecosistémicos asociados a la pesca provistos por las Áreas Marinas Protegidas en Colombia. Documentos CEDE, 2014(3), 1-57.

Dixon, J. A.; Scura, L. F.; Carpenter, R. J.; Sherman, P. B. (1994). Economic analysis of environmental impacts. Washington, D.C.: World Bank Group.

Echeverría, B. J. (2004). Selección de escenarios de emisiones de gases de efecto invernadero para desarrollar escenarios de cambio climático en Centroamérica. Documento Técnico, CRRH-UCR-CIGEFI-AIACCLA06. San José, Costa Rica.

Forest, C. E., Stone, P. H., \& Sokolov, A. P. (2006). Estimated PDFs of climate system properties including natural and anthropogenic forcings. Geophysical Research Letters, 33(1), L01705.

Freeman, A. M., Herriges, J. A., \& Kling, C. L. (2014). The Measurement of Environmental and Resource Values. Theory and Methods. Resources for the Future. Third Edition. New York, NY: Routledge.

García-Cuellar, J. A., Arreguin-Sánchez, F., Hernández, S., \& Lluch-Cota, D. B. (2004). Impacto ecológico de la industria petrolera en la sonda de Campeche, México, tras tres décadas de actividad: Una revisión. Interciencia, 29 (6), 311-319.

Gómez, A. M. (2017). Valoración económica de los servicios ecosistémicos proporcionados por un ecosistema costero-marítimo aplicando la metodología Amuvan: caso implementación costa de la Safor. Comunidad valenciana. (Tesis de maestría). Universitat Politècnica de València, España.

Graham, E. (2008). Man's impact on the coastline. Journal of Iberian Geology, 34(2), 167-190.

Hay, J., \& Mimura, N. (2006). Supporting climate change vulnerability and adaptation assessments in the AsiaPacific region: an example of sustainability science. Sustainability Science, 1(1), 23-35.

Hennessy, K., Fitzharris, B., Bates, B. C., Harvey, N., Howden, S. M., Hughes, L., ... \& Warrick, R. (2007). Australia and New Zealand. In M. L. Parry, O. F. Canziana, J. P. Palitikof, P. J. van der Linder, \& C. E Hanson (Eds.), Climate Change 2007 - Impacts, Adaptation and Vulnerability: Working Group II Contribution to the Fourth Assessment Report of the IPCC (pp. 507-540). Cambridge, UK: Cambridge University Press.

Intergubernamental Panel on Climate Change [IPCC] (2001). Cambio Climático 2001: Informe de síntesis. Resumen para responsables de politica. Recuperado 
de https://www.ipcc.ch/pdf/glossary/tar-ipcc-termssp.pdf.

Jonkman, S. N., Stive, M. J., \& Vrijling, J. K. (2005). New Orleans is a lesson for the Dutch. Journal of Coastal Research, 21, xi-xii+1191.

Knutti, R., Stocker, T. F., Joos, F., \& Plattner, G. K. (2002). Constraints on radioactive forcing and future climate change from observations and climate model ensembles. Nature, 416, 719-723.

Kuhfuss, L., Rey-Valette, H., Sourisseau, E., Heurtefeux, H., \& Rufray, X. (2016). Evaluating the impacts of sea level rise on coastal wetlands in LanguedocRoussillon, France. Environmental Science \& Policy, 59, 26-34.

Lizano, O. G. (1997). Las mareas extraordinarias de 1997 en la costa Pacífica de Costa Rica. Tópicos Meteorológicos y Oceanográficos, 4(2), 169-179.

Lizano, O. G., \& Salas, D. M. (2001). Variaciones geomorfológicas en los últimos 50 años de la Isla Damas, Quepos, Costa Rica. Revista de Biología Tropical, 49(2), 171-177.

Lotze, H. K., Lenihan, H. S., Bourque, B. J., Bradbury, R. H., Cooke, R. G., Kay, M. C., ... \& Jackson, J. B. C. (2006). Depletion, degradation and recovery potential of estuaries and coastal seas. Science, 312, 1806-1809.

Maldonado, J. H., \& Cuervo-Sánchez, R. (2016). Valoración económica del Parque Nacional Natural Corales de Profundidad. Boletín de Investigaciones Marinas y Costeras, 45(1), 99-121.

Meinshausen, M. (2006). What does a $2^{\circ} \mathrm{C}$ target mean for greenhouse gas concentrations? A brief analysis based on multi-gas emission pathways and several climate sensitivity uncertainty estimates. In H. J. Schellnhuber, W. Cramer, N. Nakicenovic, T. Wigley, \& G. Yohe (Eds.), Avoiding Dangerous Climate Change (pp.265-280). Cambridge, UK: Cambridge University Press.

Melo, S., Riveros, L., Romero, G., Álvarez, A., Diaz, C., \& Calderón, S. (2017). Efectos económicos de futuras sequías en Colombia: Estimación a partir del Fenómeno El Niño 2015. Archivos de Economía, 466, 1-34.

Moreno-Díaz, M. L. (2009). Propuesta metodológica para valorar el impacto de las actividades económicas en áreas costeras. Revista Iberoamericana de Economía Ecológica, 11, 29-38.

Moreno-Díaz, M. L. (2012). Actividades socioeconómicas en el Parque Nacional Isla del Coco, Costa Rica y posibles efectos de la variabilidad climática. Revista de Biología Tropical, 60(Supplement 3), 113-129.
Moreno-Díaz, M. L. (2015). Valoración del impacto socioeconómico de la variabilidad climática en pesca y turismo. Revista Ciencias Sociales, 147(1), 73-83.

Munday, P. L., Leis, J. M., Loudh, J. M., Paris, C. B., Kingsford, M. J., Berumen, M. L., \& Lambrechts, J. (2009). Climate change and coral reef connectivity. Coral Reefs, 28, 379-395.

Murphy, J. M., Sexton, D. M. H., Barnett, D. N., Jones, G. S., Webb, M. J., Collins, M., \& Stainforth, D. A. (2004). Quantification of modelling uncertainties in a large ensemble of climate change simulations. Nature, 430, 768-772.

Nicholls, R. J., Wong, P. P., Burkett, V. R., Codignotto, J. O., \& Hay, J. E. (2007). Coastal systems and lowlying areas. In M. L. Parry, O. F. Canziana, J. P. Palitikof, P. J. van der Linder, \& C. E Hanson (Eds.), Climate Change 2007 - Impacts, Adaptation and Vulnerability: Working Group II Contribution to the Fourth Assessment Report of the IPCC (pp. 315-356). Cambridge, UK: Cambridge University Press.

Ong, J. E. (2001). Vulnerability of Malaysia to sea level change. Proceedings of the APN/SURVAS/LOICZ Joint Conference on Coastal Impacts of Climate Change and Adaptation in the Asia - Pacific Region, 14-16th November 2000, Kobe, Japan, Asia Pacific Network for Global Change Research, 89-93.

Pearce, D., \& Turner, K. (1995). Economía de los recursos naturales y del medio ambiente. Madrid, España: Ediciones Colegio de Economistas de Madrid.

Pirazzoli, P. A., Regnauld, H., \& Lemasson, L. (2004). Changes in storminess and surges in western France during the last century. Marine Geology, 210, 307-323.

Ranasinghe, R., McLoughlin, R., Short, A., \& Symonds, G. (2004). The Southern Oscillation Index, wave climate, and beach rotation. Marine Geology, 204(34), 273-287.

Raschky, P. A. (2008). Institutions and the losses from natural disasters. Natural Hazards and Earth System Sciences. European Geosciences Union, 8(4), 627-634

Regnauld, H., Pirazzoli, P. A., Morvan, G., \& Ruz, M. (2004). Impacts of storms and evolution of the coastline in western France. Marine Geology, 210(1-4), 325-337.

Roebeling, P. C., Costa, L., Magalhães-Filho, L., \& Tekken, V. (2013). Ecosystem service value losses from coastal erosion in Europe: historical trends and future projections. Journal of Coastal Conservation, 17(3), 389-395.

Rogers, K., \& Saintilan, N. (2008). Relationships between surface elevation and groundwater in mangrove 
forests of southeast Australia. Journal of Coastal Research, 24(1), 63-69.

Scavia, D., Field, J. C., Boesch, D. F., Buddemeier, R., Cayan, D. R., Burkett, V., ...Titus, J. G. (2002). Climate change impacts on U.S. coastal and marine ecosystems. Estuaries, 25, 149-164.

Schallenberg, M., Hall, C. J., \& Burns, C. W. (2003). Consequences of climate-induced salinity increases on zooplankton abundance and diversity in coastal lakes. Marine Ecology Progress Series, 251, 181-189.

Sharp, B., \& Batstone, C. (2008). Neoclassical framework for optimizing the value of marine resources. In M. Patterson \& B. Glavovic (Eds.), Ecological Economics of the Oceans and Coasts (pp. 95-117). Cheltenham, UK: Edward Elgar Publishing.

Timmermann, A., An, S. I., Kug, J. S., Jin, F. F., Cai, W. Capotondi, A., ... \& Stein, K. (2018). El Niño-southern oscillation complexity. Nature, 559(7715), 535.

Tol, R. S. (2007). The double trade-off between adaptation and mitigation for sea level rise: an application of FUND. Mitigation and Adaptation Strategies for Global Change, 12(5), 741-753.
Valdés, J., \& Sifeddine, A. (2009). Composición elemental y contenido de metales en sedimentos marinos de la bahía Mejillones del Sur, Chile: evaluación ambiental de la zona costera. Latin American Journal of Aquatic Research, 37(2), 131-141.

Viles, H. A., \& Goudie, A. S. (2003). Interannual, decadal and multidecadal scale climatic variability and geomorphology. Earth-Science Reviews, 61(1-2), 105-131.

Walsh, R., Loomis, L., \& Gillman, R. (1984). Valuing option, existence, and bequest demands for wilderness. Land Economics, 60(1), 14-29.

Wigley, T. M. L., \& Raper, S. C. B. (2001). Interpretation of high projections for global-mean warming, Science, 293, 451-454.

Wilson, M., \& Liu, S. (2008). Non-market value of ecosystem services provided by coastal and nearshore marine systems. In M. Patterson \& B. Glavovic (Eds.), Ecological Economics of the Oceans and Coasts (pp. 119-139). Cheltenham, UK: Edward Elgar Publishing.

Zweig, R. (1998). Sustainable Aquaculture: Seizing Opportunities to Meet Global Demand. Agriculture Technology Notes, 22, 1-4. 